\title{
Bayesian risk maps for Schistosoma mansoni and hookworm mono-infections in a setting where both parasites co-exist
}

\author{
Giovanna Raso ${ }^{1,2}$, Penelope Vounatsou ${ }^{3}$, Donald P. McManus², Jürg Utzinger ${ }^{3}$ \\ ${ }^{1}$ Division of Epidemiology and Social Medicine, School of Population Health, The University of \\ Queensland, Herston Road, Brisbane, QLD 4006, Australia; ${ }^{2}$ Molecular Parasitology Laboratory, The \\ Queensland Institute of Medical Research, 300 Herston Road, Brisbane, QLD 4006, Australia; \\ ${ }^{3}$ Department of Public Health and Epidemiology, Swiss Tropical Institute, P.O. Box, CH-4002 Basel, \\ Switzerland
}

\begin{abstract}
There is growing interest in the use of Bayesian geostatistical models for predicting the spatial distribution of parasitic infections, including hookworm, Schistosoma mansoni and co-infections with both parasites. The aim of this study was to predict the spatial distribution of mono-infections with either hookworm or $S$. mansoni in a setting where both parasites co-exist. School-based cross-sectional parasitological and questionnaire surveys were carried out in 57 rural schools in the Man region, western Côte d'Ivoire. A single stool specimen was obtained from each schoolchild attending grades 3-5. Stool specimens were processed by the Kato-Katz technique and an ether concentration method and examined for the presence of hookworm and S. mansoni eggs. The combined results from the two diagnostic approaches were considered for the infection status of each child. Demographic data (i.e. age and sex) were obtained from readily available school registries. Each child's socio-economic status was estimated, using the questionnaire data following a household-based asset approach. Environmental data were extracted from satellite imagery. The different data sources were incorporated into a geographical information system. Finally, a Bayesian spatial multinomial regression model was constructed and the spatial patterns of $S$. mansoni and hookworm mono-infections were investigated using Bayesian kriging. Our approach facilitated the production of smooth risk maps for hookworm and S. mansoni mono-infections that can be utilized for targeting control interventions. We argue that in settings where $S$. mansoni and hookworm co-exist and control efforts are under way, there is a need for both mono- and co-infection risk maps to enhance the cost-effectiveness of control programmes.
\end{abstract}

Keywords: Hookworm, schistosomiasis, Schistosoma mansoni, geographical information system, risk mapping, coinfection, Bayesian geostatistics, Côte d'Ivoire.

\section{Introduction}

Bayesian geostatistical approaches are increasingly utilized for mapping and predicting the risk of malaria and the so-called neglected tropical diseases (Basáñez et al., 2004; Gemperli et al., 2004; Raso et al., 2005a; Clements et al., 2006; Gosoniu et al., 2006; Beck-Wörner et al., 2007; Diggle et al.,

Corresponding author:

Giovanna Raso

School of Population Health

The University of Queensland

Herston Road, Brisbane, QLD 4006, Australia

Tel. +61 7336 55447; Fax: +61 733655599

E-mail: g.raso@uq.edu.au
2007). Thus far, the majority of investigations have focussed on the risk of a single parasitic infection. The generated risk maps are useful for health policy makers to readily target control interventions. For example, Bayesian geostatistical methods have been employed to predict the spatial distribution of Schistosoma mansoni and S. haematobium in Tanzania, and the national schistosomiasis control programme takes advantage of this knowledge-base for prioritizing the large-scale administration of praziquantel (Clements et al., 2006).

It is important to note that malaria and the neglected tropical diseases are co-endemic over large parts of the developing world (Raso et al., 2004a; 
Brooker et al., 2006; Brooker and Utzinger, 2007). The issue of co-endemicity is of considerable public health importance and offers opportunities for combined control approaches. For example, the World Health Organization (WHO) recommends that in high burden areas of schistosomiasis and soil-transmitted helminthiasis, praziquantel and albendazole/mebendazole should be regularly administered to school-aged children and other high-risk groups (WHO, 2002). Implementation of this recommendation has been facilitated by the creation of the Partners for Parasite Control (PPC; http:// www.who.int/wormcontrol), the Schistosomiasis Control Initiative (SCI; http://www.schisto.org), and funding through the Bill and Melinda Gates Foundation (Fenwick, 2006; Kabatereine et al., 2006). It has also been suggested that the control of several neglected tropical diseases can be achieved by administration of just four drugs, which has been portrayed as one of the most cost-effective interventions in public health (Molyneux et al., 2005; Brady et al., 2006; Hotez et al., 2006, 2007). Mass drug administration without prior diagnosis, however, means that a certain proportion of individuals will be given drugs unnecessarily. The lower the prevalence of two infections in the same area, the lower the risk of a co-infection, and hence the higher the proportion of treating people unnecessarily.

Predicting the spatial distribution of co-infections represents an important step forward not only with regard to priority setting but also to enhancing the cost-effectiveness of integrating control programmes that target multiple diseases simultaneously. We have recently presented an approach for predicting the spatial distribution of $S$. mansoni-hookworm co-infections using Bayesian multinomial regression models (Raso et al., 2006b). Such multinomial models can be readily adapted to other co-infections or multiple species parasitic infections. Although no other studies employing these multinomial models have been presented, it is conceivable that control programmes will make use of such models in the future.

Integrated control programmes targeting several diseases simultaneously will reduce the extent of coendemicity over time. Hence mono-infections (for a definition see Box 1) will gain in relative importance when compared to co-infections. This issue is illustrated in Figure 1. If, for example, a cross-sectional survey, carried out in an area endemic for both S. mansoni and hookworm, reveals an infection prevalence of each parasite of $50 \%$, the estimated co-infection prevalence would be $25 \%$, and $25 \%$ of the individuals would be free of any infection, assuming independence of the infections. If praziquantel and albendazole/mebendazole were administered to all individuals, $25 \%$ of the people would be treated unnecessarily with both drugs. Now, suppose that regular rounds of chemotherapy have halved the prevalence of $S$. mansoni and hookworm in that area. Co-infections would then have become much less prominent, i.e. the estimated coinfection prevalence is $6.25 \%$. The respective estimated mono-infection prevalence of $S$. mansoni and hookworm would have increased in relative importance, namely $18.75 \%$ each.

On the basis of the conceptual framework presented in Figure 1, we argue that there is a need not only to predict the spatial distribution of single infections and co-infections, but indeed also monoinfections. Here, we present the risk prediction of S. mansoni and hookworm mono-infections and discuss the practical implications of such risk maps

Box 1. Definition of selected terms used in this article.

Co-endemicity: An area where two investigated diseases are simultaneously endemic.

Co-infection: This applies to individuals harbouring two infections simultaneously.

Single infection: When individuals harbour an infection and other infections that might be present are not considered.

Mono-infection: Individuals harbour only one infection, with regard to other investigated endemic infections. 
for the targeted control of schistosomiasis and soiltransmitted helminthiases.

\section{Materials and methods}

\section{Study area and population}

The study was carried out between October 2001 and July 2002 in the Man region, western Côte d'Ivoire. As has been documented before, both $S$. mansoni and hookworm infections are common in this setting among schoolchildren and entire communities (Utzinger et al., 1999; Keiser et al., 2002; Raso et al., 2004a, 2005b). Our study population consisted of schoolchildren aged 6 to 16 years who attended grades 3-5 in 57 rural schools.

Demographic, socio-economic and parasitological data

Demographic data, i.e. name, age and sex of schoolchildren were readily available from educa- tion registries. The socio-economic status of households where schoolchildren resided was assessed by means of a questionnaire administered to schoolchildren. The questionnaire included a list of 10 assets of ownership (e.g. possession of a radio) and two specific items on household characteristics (e.g. walls constructed with bricks). We used an assetbased approach to stratify schoolchildren into wealth quintiles (Filmer and Pritchett, 2001).

Parasitological data were obtained from a crosssectional survey that focused on the infection status of the schoolchildren with S. mansoni and hookworm. All schoolchildren were invited to submit one stool specimen. Two different diagnostic approaches were employed as follows. First, a single $42 \mathrm{mg}$ Kato-Katz thick smear was prepared on microscope slides (Katz et al., 1972). After a clearing time of $30-45 \mathrm{~min}$, the slides were examined under a light microscope at low magnification by experienced laboratory technicians. Eggs of $S$. mansoni and hookworm were counted and recorded separately. Second, a 1-2 g portion of stool was

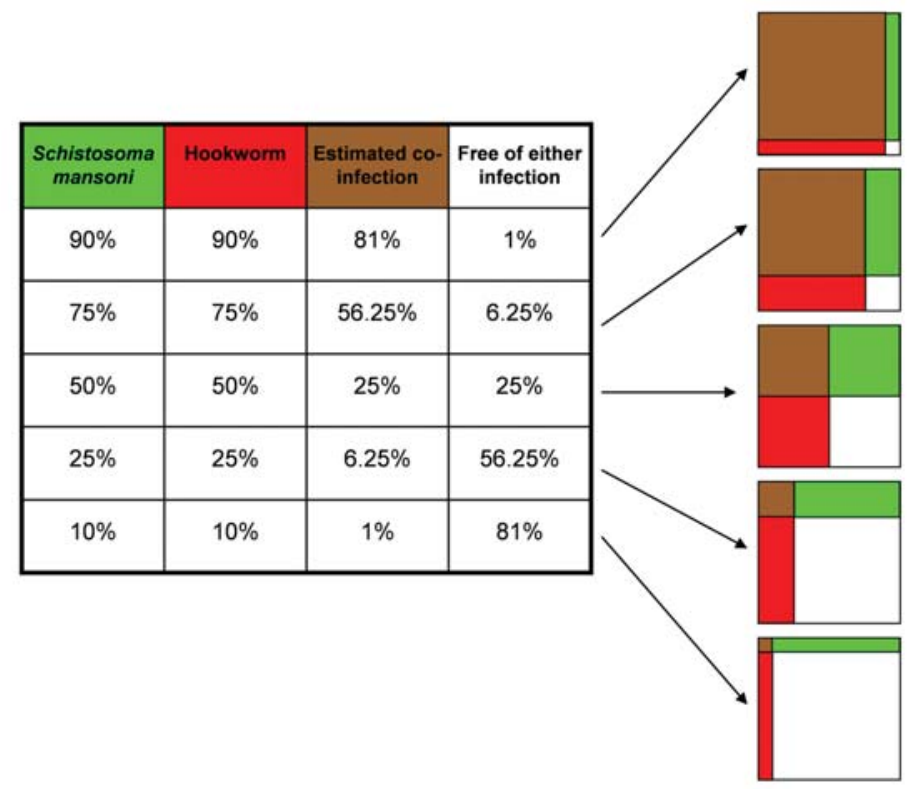

Fig. 1. Conceptual framework for an area co-endemic for S. mansoni and hookworm, where several rounds of mass drug administration take place. It highlights that treating all individuals in a co-endemic area with praziquantel and albendazole/mebendazole decreases the prevalence of $S$. mansoni and hookworm and, consequently, also the prevalence of co-infection. As a result, an ever growing proportion of individuals will be treated unnecessarily with one or even both drugs. 
fixed in $10 \mathrm{ml}$ solution of sodium-acetic acid-formalin (SAF). The fixed stool samples were processed using an ether concentration method, and analyzed under a light microscope for helminth eggs and intestinal protozoa (Allen and Ridley, 1970; Marti and Escher, 1990). Only the results pertaining to S. mansoni and hookworm were further analyzed here. A child was considered positive for an infection with $S$. mansoni or hookworm if at least one egg was detected either on the Kato-Katz thick smear, or the SAF-preserved stool sample following the ether-concentration method. The pooled parasitological data (combined Kato-Katz and SAFether-concentration readings) were considered the 'composite reference standard' and used in all subsequent analyses.

\section{Environmental data}

Geographical coordinates of each school were obtained using a hand-held global positioning system (GPS; Thales Navigation, Santa Clara, CA, USA). Land cover types were derived from the Advanced Very High Resolution Radiometer (AVHRR) satellite, U.S. Geological Survey (USGS) Africa Land Cover Characteristics Database version 2: Africa Seasonal Land Cover Regions, USGS Earth Resources Observation System (EROS) Data Centre at $1 \times 1 \mathrm{~km}$ spatial resolution. The interpolated digital elevation model (DEM) was obtained from the USGS EROS Data Center (http://lpdaac.usgs.gov/gtopo30/gtopo_links.asp).

\section{Ethical considerations and treatment}

The study protocol was approved by the institutional review boards of the Swiss Tropical Institute (Basel, Switzerland) and the Centre Suisse de Recherches Scientifiques (Abidjan, Côte d'Ivoire). Ethical clearance was granted by the Ministry of Public Health in Côte d'Ivoire. Written informed consent was obtained from the district education officers after explaining the aims, procedures and potential risks and benefits of the current study.
Education officers informed school directors about the study and detailed information was provided by our research team the day preceding the parasitological survey.

Children infected with $S$. mansoni were given a single $40 \mathrm{mg} / \mathrm{kg}$ oral dose of praziquantel, and children infected with hookworm or other soil-transmitted helminths were administered a single $400 \mathrm{mg}$ oral dose of albendazole, according to recommendations by WHO (WHO, 2002).

\section{Statistical analysis and model specification}

Schoolchildren were classified into two age groups (group 1: children aged 6-10 years; group 2: children aged 11-16 years). An outcome variable was created with the following four categories: (1) co-infection with S. mansoni and hookworm, (2) mono-infection with S. mansoni, (3) mono-infection with hookworm, and (4) no infection.

The Pearson's $\chi^{2}$-test was used to assess association between age and sex with $S$. mansoni and hookworm, single and mono-infections. Bivariate multinomial models were also fitted for each available demographic, socio-economic and environmental covariate on the 4-category outcome variable. The above analyses were carried out in STATA version 9.2 (Stata Corporation, College Station, TX, USA). Significant covariates by the likelihood ratio test (LRT) at 20\% significance level were built into a Bayesian spatial multinomial regression model for a mono-infection with either $S$. mansoni or hookworm, and for a S. mansoni-hookworm co-infection (Raso et al., 2006b). The spatial analysis was done in WinBUGS version 1.4.2 (Imperial College \& Medical Research Council, London, UK).

Let $Y_{i j k}$ and $p_{i j k}$ be the infection status and probability of co-infection $(k=1)$, a $S$. mansoni monoinfection $(k=2)$, a hookworm mono-infection $(k=$ $3)$, and no infection $(k=4)$, of schoolchild $j$ in village $i$. We assumed that $Y_{i j k}$ arises from a multinomial distribution, i.e.

$$
\left(Y_{i j 1}, Y_{i j 2}, Y_{i j 3}, Y_{i j 4}\right) \sim M u l t\left(1, p_{i j 1}, p_{i j 2}, p_{i j 3}, p_{i j 4}\right)
$$


and we modeled the influence of covariates $\underline{X}_{i j k}$ and village-specific random effects $\phi_{i k}$ on the

$$
\log \left(\frac{p_{i j k}}{p_{i j 4}}\right), k=1,2,3 \text {, as } \log \left(\frac{p_{i j k}}{p_{i j 4}}\right)=\underline{X}_{i j}^{T} \underline{\beta}+\phi_{i k}
$$

where $\underline{\beta}=\left\{\underline{\beta}_{k}\right\}_{k=1,2,3}=\left(\underline{\beta_{1}}, \underline{\beta_{2}}, \underline{\beta_{3}}\right)$ is the vector of regression coefficients corresponding to the multinomial categories. $\frac{p_{i j k}}{p_{i j 4}}$ is the risk ratio (RR) of the infection status with regard to no infection. The spatial correlation structure was introduced on $\phi_{k}=\left(\phi_{1 k}, \phi_{2 k}, \ldots \phi_{N k}\right)^{T}$ that has a multivariate normal distribution, $\bar{\phi}_{k} \sim \operatorname{MVN}\left(\underline{0}, \sum_{k}\right)$, with variancecovariance matrix $\sum_{k}$. Further, we assumed an isotropic spatial process, i.e. $\sum_{i r k}=\sigma_{k}^{2} \exp \left(-u_{k} d_{i r}\right)$.

$d_{i r}$ is the Euclidean distance between villages $i$ and $r, \sigma_{k}^{2}$ is the geographic variability and $u_{k}$ is the smoothing parameter that controls the rate of correlation decay with increasing distance. The minimum distance at which spatial correlation between locations is below $5 \%$ for each category $k$ was calculated as $3 / u_{k}$ and expressed in meters.

We adopted prior distributions for the model parameters and choose vague Normal prior distributions for the $\beta$ parameters with large variances (i.e. 10,000), inverse gamma priors for $\left\{\sigma_{k}^{2}\right\}_{k}=1,2,3$ and uniform priors for $\left\{u_{k}\right\}_{k}=1,2,3$.
We employed Markov chain Monte Carlo (MCMC) simulation to estimate the model parameters (Gelfand and Smith, 1990) and ran a single chain sampler with a burn-in of 5000 iterations. Convergence of the model was assessed by inspecting the ergodic averages of selected model parameters. Covariates from the multivariate model were used to generate smooth risk maps of mono-infections, using Bayesian kriging (Diggle et al., 1998).

\section{Results}

\section{Study cohort and parasitological findings}

The final study cohort, i.e. schoolchildren who had complete parasitological and questionnaire data, consisted of 3578 children from 56 schools. There were significantly more boys $(\mathrm{n}=2162)$ than girls $(\mathrm{n}=1416 ; \mathrm{P}<0.001)$. With regard to age groups, there were 1956 children in the age group 610 years $(54.7 \%)$ and the remaining 1622 children were aged 11-16 years.

The cross-sectional parasitological survey found that 1167 children $(32.6 \%)$ were free of $S$. mansoni and hookworm infections, 869 children $(24.3 \%)$ had a mono-infection with hookworm, 862 children

Table 1. Comparison of demographic factors associated with S. mansoni and hookworm single and mono-infections.

\begin{tabular}{|c|c|c|c|c|c|c|c|c|}
\hline \multirow[t]{2}{*}{ Feature } & \multicolumn{4}{|c|}{ Schistosoma mansoni } & \multicolumn{4}{|c|}{ Hookworm } \\
\hline & $\begin{array}{c}\text { Number } \\
\text { of children }(\%)\end{array}$ & $\begin{array}{c}\text { School range } \\
(\%)\end{array}$ & $\chi^{2}$ & P-value & $\begin{array}{c}\text { Number } \\
\text { of children }(\%)\end{array}$ & $\begin{array}{c}\text { School range } \\
(\%)\end{array}$ & $x^{2}$ & P-value \\
\hline \multicolumn{9}{|c|}{ Single infection } \\
\hline \multicolumn{9}{|c|}{ Sex } \\
\hline Male & $980(63.6)$ & $0-96.4$ & & & $1079(69.7)$ & $9.5-87.2$ & & \\
\hline Female & $562(36.4)$ & $0-93.3$ & 11.09 & 0.001 & $470(30.3)$ & $9.1-65.0$ & 97.38 & $<0.001$ \\
\hline \multicolumn{9}{|c|}{ Age (years) } \\
\hline 6-10 & $791(51.3)$ & $0-95.0$ & & & 809 (52.2) & $0-73.3$ & & \\
\hline 11-16 & $751(48.7)$ & $0-96.3$ & 12.42 & $<0.001$ & $740(47.8)$ & $7.7-86.7$ & 6.56 & 0.010 \\
\hline \multicolumn{9}{|c|}{ Mono-infection } \\
\hline \multicolumn{9}{|c|}{ Sex } \\
\hline Male & $489(56.7)$ & $0-69.0$ & & & $588(67.7)$ & $0-72.3$ & & \\
\hline Female & $373(43.3)$ & $0-76.0$ & 6.49 & 0.011 & $281(32.3)$ & $0-55.9$ & 25.15 & $<0.001$ \\
\hline \multicolumn{9}{|c|}{ Age (years) } \\
\hline 6-10 & $450(52.2)$ & $0-61.4$ & & & $468(53.9)$ & $0-71.2$ & & \\
\hline $11-16$ & $412(47.8)$ & $0-76.9$ & 2.78 & 0.095 & $401(46.1)$ & $0-77.3$ & 0.31 & 0.580 \\
\hline
\end{tabular}



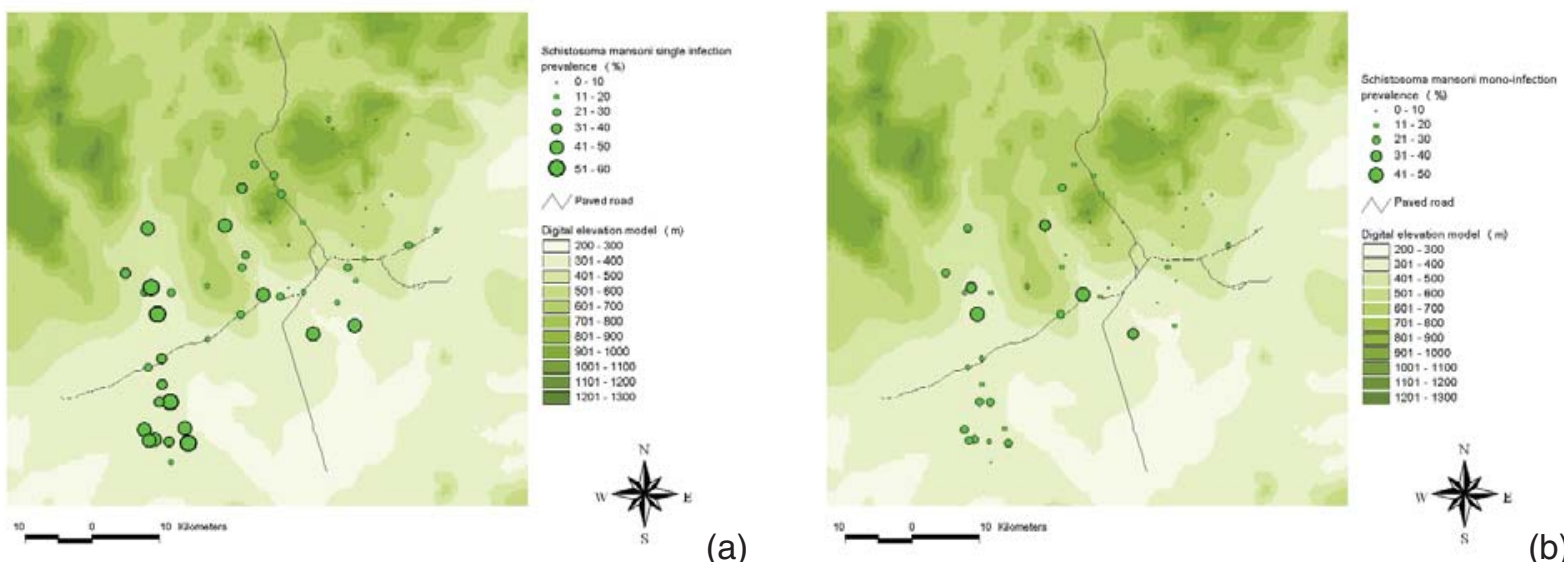

Fig. 2. Distribution of S. mansoni single infections (a) and S. mansoni mono-infections (b) among schoolchildren at the unit of school in the Man region, western Côte d'Ivoire.
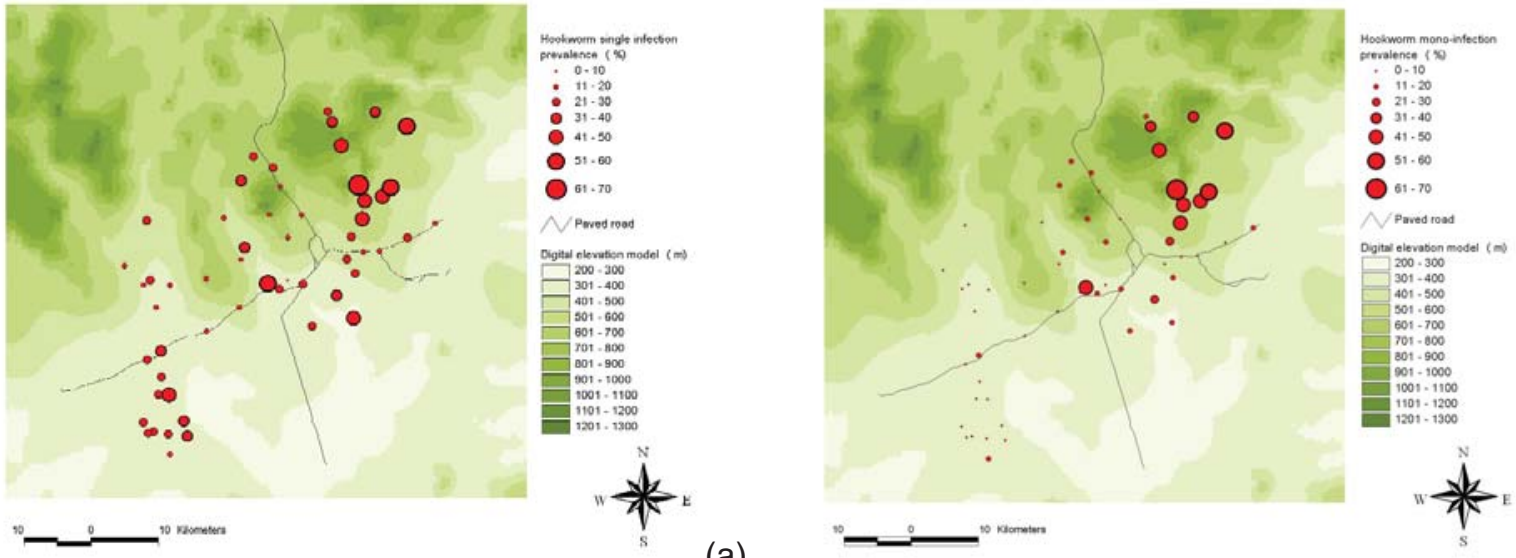

(a)

Fig. 3. Distribution of hookworm single infections (a) and hookworm mono-infections (b) among schoolchildren at the unit of school in the Man region, western Côte d'Ivoire.

$(24.1 \%)$ were infected only with S. mansoni, and 680 children $(19.0 \%)$ had a co-infection with both parasites. At the unit of the school, the prevalence of $S$. mansoni-hookworm co-infection ranged from $0 \%$ to $60.0 \%$, the prevalence of $S$. mansoni monoinfection ranged from $0 \%$ to $63.9 \%$, and monoinfections with hookworm ranged from $0 \%$ to $65.7 \%$. If single infections were considered, 1542 children $(43.1 \%)$ had a $S$. mansoni infection and 1549 children $(43.3 \%)$ had a hookworm infection. Table 1 shows a comparison between S. mansoni and hookworm single-infections versus mono-infections, stratified by demographic indicators. While age and sex were significantly associated with single infections with $S$. mansoni and hookworm, age was associated neither to $S$. mansoni nor to hookworm mono-infections according to $\chi^{2}$-test.

The highest frequencies of a $S$. mansoni-hookworm co-infection were observed in the south-western part of the study area (Raso et al., 2006b). A comparison of the observed single and mono-infection prevalence of $S$. mansoni at the unit of the 
school is shown in Figures $2 \mathrm{a}$ and $2 \mathrm{~b}$. The comparison between hookworm single and mono-infection is shown in Figures $3 \mathrm{a}$ and $3 \mathrm{~b}$.

\section{Spatial analysis}

The significant covariates identified by the bivariate non-spatial multinomial regression analyses, which were sex (LRT = 109.11, P <0.001), age group (LRT $=20.07, \mathrm{P}<0.001)$, socio-economic status $(\mathrm{LRT}=$ 53.73, $\mathrm{P}<0.001)$, land cover $(\mathrm{LRT}=66.37, \mathrm{P}<0.001)$ and elevation $(\mathrm{LRT}=356.51, \mathrm{P}<0.001)$, were built into the final Bayesian spatial multinomial regression model.

The risk profiling results for the Bayesian spatial multinomial regression model have been presented in detail (Raso et al., 2006b) and revealed that boys were more likely to have a $S$. mansoni-hookworm co-infection than girls (risk ratio ratio $(\mathrm{RRR})=2.45$ ), and children aged 11-16 years were at a higher risk of a co-infection than their younger counterparts (RRR = 1.55 ). Children aged 11-16 years were not at a significantly higher risk of a hookworm mono-infection, but they were so in the case of a $S$. mansoni monoinfection $(\mathrm{RRR}=1.59)$. Boys were not significantly more likely to have a $S$. mansoni mono-infection but they were at a significantly higher risk of a hookworm mono-infection $(\mathrm{RRR}=2.02)$. Children belonging to the two poorest wealth quintiles were approximately at a 2-fold higher risk of a $S$. mansoni-hookworm coinfection when compared to children from wealthier households. Children from the poorest quintile were also at a significantly higher risk of mono-infections with either S. mansoni or hookworm compared to the least poor quintile. With regard to environmental covariates, elevation showed the strongest leverage on a S. mansoni-hookworm co-infection; children attending schools at altitudes below $400 \mathrm{~m}$ above sea level were at a 4.5 -fold higher risk than those living at altitudes of $400 \mathrm{~m}$ and above. Furthermore, children living at altitudes below $400 \mathrm{~m}$ where at a 5-fold higher risk of $S$. mansoni mono-infections, but 2 -fold lower risk of hookworm mono-infections compared to children living at altitudes $\geq 400 \mathrm{~m}$. Whilst tropical forest showed a significant association with a hookworm mono-infection, none of the land cover types investigated was significantly associated with either a $S$. mansoni mono-infection or a S. mansoni-hookworm co-infection. The range at which the spatial correlation was below $5 \%$, and hence non-significant, was $4.1 \mathrm{~km}$ for $S$. mansoni mono-infections, $1.3 \mathrm{~km}$ for hookworm mono-infections, and $3.3 \mathrm{~km}$ for a coinfection with both parasites. For comparison, the $S$. mansoni and hookworm single infection models in our previous analyses revealed spatial correlations $<5 \%$ at distances of $7.5 \mathrm{~km}$ (Raso et al., 2005a) and $1.2 \mathrm{~km}$ (Raso et al., 2006a), respectively. The geographical variability was 1.68 for $S$. mansoni monoinfections, 0.48 for hookworm mono-infections, and 1.31 for co-infections with both parasites.

\section{Prediction of S. mansoni and hookworm mono- infections}

Figure 4 a shows the predicted $S$. mansoni monoinfection prevalence. The highest prevalence of $S$. mansoni mono-infection was predicted for the central-western part of the study area. With regard to $S$. mansoni single infection, the highest prevalence was predicted in the south-western part (see Raso et al., 2005a for the respective risk map). Apart from these differences in the predicted high-risk areas, the two maps show considerable similarities.

Standard deviations of the predicted S. mansoni mono-infection prevalence became larger with increasing distance from the sampled locations, as shown in Figure 4b. The same trend had already been observed in our previous work focusing on $S$. mansoni single infection (Raso et al., 2005a).

Figure 5a shows the predicted hookworm monoinfection prevalence. There are considerable differences when compared to the previously predicted risk map of hookworm single infection (Raso et al., 2006a). Whilst the highest risk areas for a hookworm mono-infection have been predicted for the northern part of the study area and, conversely, lowrisk areas have been predicted for the southern part, no clear patterns have been found before for hook- 

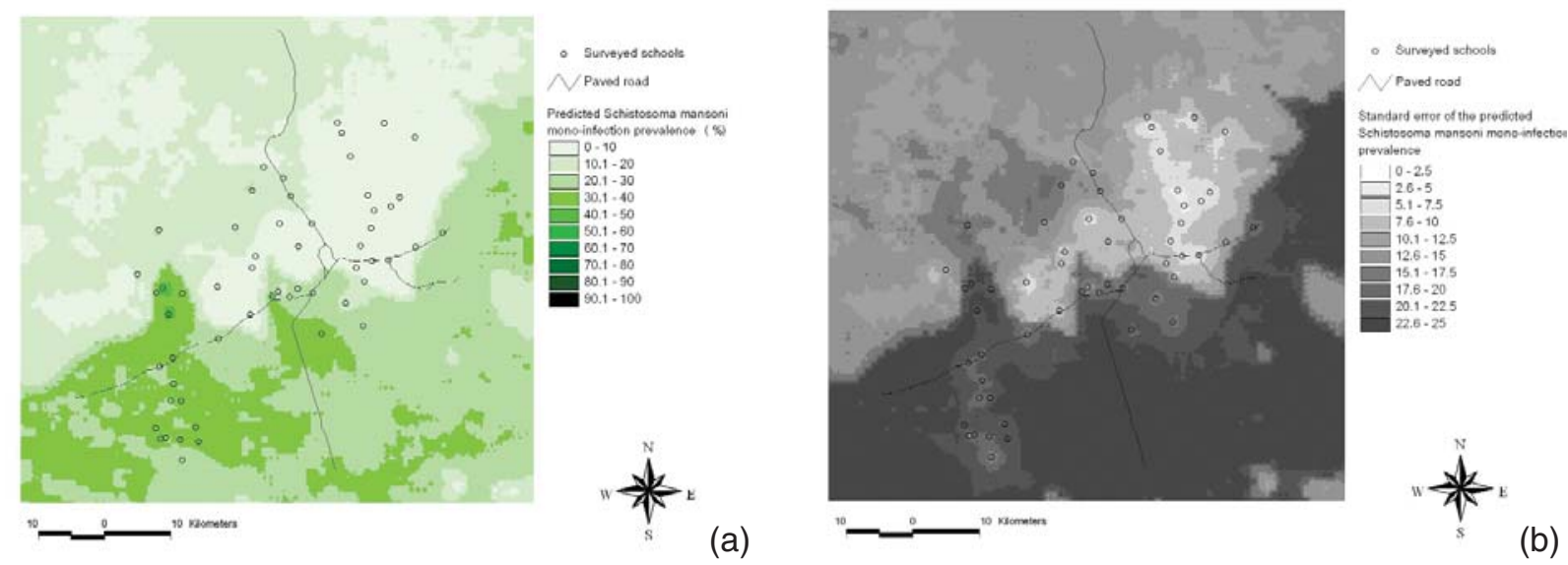

Fig. 4. Predicted S. mansoni mono-infection prevalence using Bayesian kriging based on the multinomial regression model (a) and standard error of the predicted S. mansoni mono-infection prevalence (b).
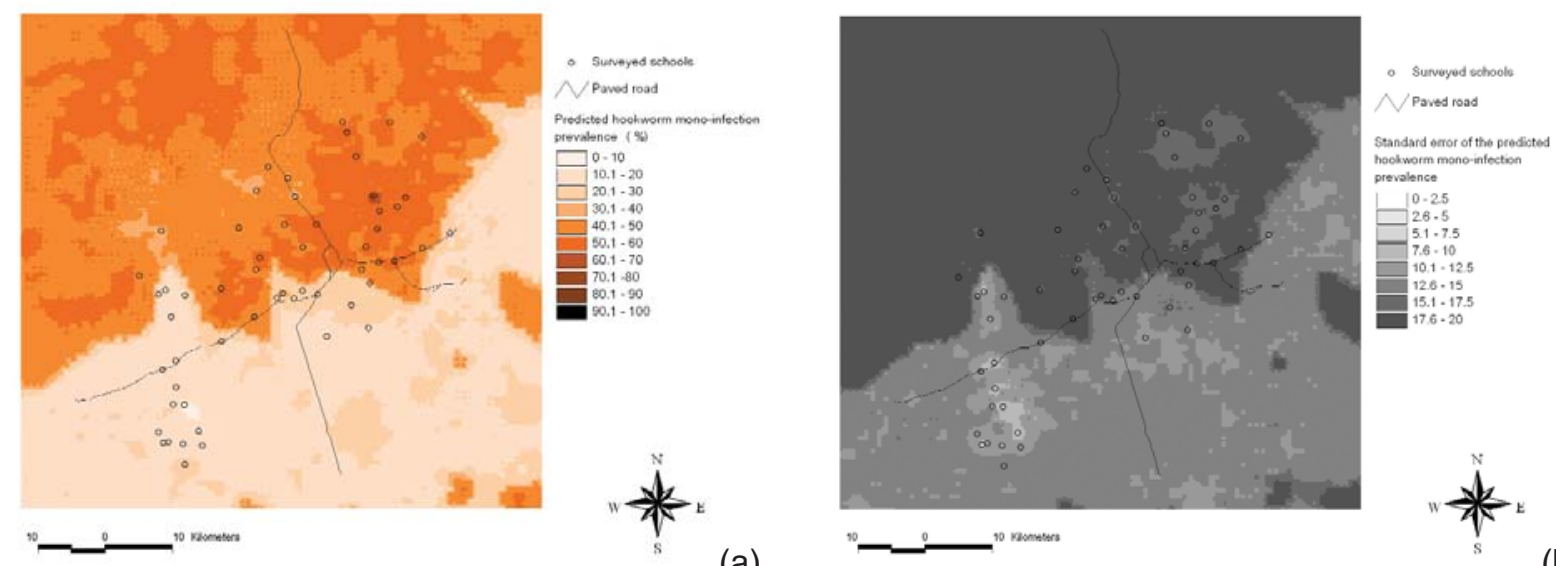

(a)

Fig. 5. Predicted hookworm mono-infection prevalence using Bayesian kriging based on the multinomial regression model (a) and standard error of the predicted hookworm mono-infection prevalence (b).

worm single infections, which showed a rather patchy distribution (Raso et al., 2006a).

Figure $5 \mathrm{~b}$ displays the standard deviations of the predicted hookworm mono-infection prevalence. Similar to $S$. mansoni (Figure $4 \mathrm{~b}$ ) we found an increasing error of the predicted prevalence with increasing distance from sampled locations. In contrast, for the hookworm single infection risk map the standard error was rather patchy in accordance with the predicted prevalence of a single hookworm infection (Raso et al., 2006a).

\section{Discussion}

There is growing recognition of the considerable geographical overlap of the "big three" (malaria, HIV/AIDS and tuberculosis) with the so-called neglected tropical diseases (e.g. schistosomiasis and soil-transmitted helminthiases) in the developing world, and the prospect and challenges for integrated control have been discussed (Hotez et al., 2006; Lammie et al., 2006; Utzinger and de Savigny, 2006; Kolaczinski et al., 2007). Fortunately, we are now 
witnessing how this growing recognition is about to translate into increased political and financial commitment to simultaneously target control interventions against multiple diseases concurrently. Coendemicity has been emphasised as one of the key features that should allow cost-effectiveness to be enhanced (Brady et al., 2006; Brooker and Utzinger, 2007). Against this background, emphasis has been placed on targeting individuals living in areas where multiple neglected tropical diseases co-exist, since these individuals are at an elevated risk of co-infections, and hence likely to suffer from co-morbidity (Ezeamama et al., 2005). Yet, far less attention has been given to individuals living in co-endemic areas who harbour only one of the targeted infections.

In Figure 1 we provide a conceptual framework that shows how the likelihood of co-infection with two parasites actually decreases as a result of multiple rounds of mass drug administration. This strategy, also recently coined preventive chemotherapy, is being promoted as one of the best advances in tropical public health (Molyneux et al., 2005; Hotez et al., 2006, 2007; WHO, 2006). Hence, the relative importance of mono-infections in settings were multiple species parasitic infections co-exist, will gain in prominence. This issue has been exemplified here with an emphasis on mono-infections with $S$. mansoni and hookworm in the Man region, western Côte d'Ivoire.

Two methodological shortcomings are important to note. First, the diagnostic sensitivity of a single Kato-Katz thick smear is low due to significant dayto-day and intra-specimen variation (Engels et al., 1996; Utzinger et al., 2001; Booth et al., 2003). Since we combined the results from the Kato-Katz technique and the ether concentration method, the sensitivity was likely enhanced, but it is conceivable that the observed hookworm and S. mansoni prevalences were still considerably underestimated (Raso et al., 2006b). Light infections are particularly prone to be missed (Utzinger et al., 2001; Raso et al., 2004b), and hence the respective bias is likely to be elevated in areas characterized by low infection intensities. Second, only schoolchildren were select- ed for this study. Consequently, there is a sampling bias related to non-enrolled school-aged children, which might lead to an underestimation of the 'true' infection prevalence and intensity. In fact, our results emphasise that schoolchildren from the poorer households have a higher burden of parasitic infections and it is probable that non-enrolled school-aged children are at a higher risk for single and multiple parasitic infections, since these nonenrolled children most likely belong to the poorest segments of the population (Filmer and Pritchett, 2001; Raso et al., 2005b).

Bayesian approaches via MCMC algorithms offer flexibility when fitting rather complex models, and hence provide computational advantages over classical frequentist methods. The Bayesian framework offers the opportunity to model spatial dependence in a hierarchical fashion by introducing area- or location- (or site-) specific random effects (Kazembe et al., 2007). In geostatistical models spatial correlation is introduced in the correlation matrix of the location-specific random effects which model a latent Gaussian spatial process (Cressie, 1993; Diggle et al., 1998). In this article, we used a Bayesian formulation of a multinomial regression model for geostatistical data that has been developed previously for the prediction of $S$. mansonihookworm co-infections (Raso et al., 2006b). In contrast to the present application that describes a spatial analysis with multinomial outcome including mono- and co-infections with S. mansoni and hookworm, previous studies have focused on the application of univariate outcomes, i.e. prevalence of a single infection. In summary, we found that 11 to 16-year-old children belonging to the poorest households and living at elevations below $400 \mathrm{~m}$, were at the highest risk of a $S$. mansoni mono-infection. With regard to hookworm mono-infections, boys from the poorest households living at elevations above $400 \mathrm{~m}$ on land classified as tropical forest were at highest risk. Our Bayesian geostatistical modelling framework allowed drawing of risk maps of S. mansoni and hookworm mono-infections. Importantly, these risk maps showed distinct differ- 
ences when compared to risk maps emphasising S. mansoni and hookworm single infection prevalence in the same study area (Raso et al., 2005a, 2006a).

The importance of predicting a mono-infection with a specific parasite is clearly shown in the case of the hookworm mono-infection risk map, which showed a distinctively different spatial pattern when compared to the single infection risk map that has been presented previously (Raso et al., 2006a). In fact, the hookworm single infection risk map showed only marginal geographical variability, as the hookworm prevalence showed a patchy distribution across the Man region. In view of these spatial patterns, we concluded that classical epidemiological risk factors (e.g. age, sex and socio-economic status) might represent more useful predictors for targeting chemotherapeutic interventions against hookworm infections at this small-to-moderate spatial scale (our study area covered a surface area of approximately 40 x $60 \mathrm{~km}$ ). However, the generated risk map for a hookworm mono-infection, as presented here, shows a distinct risk profile with the highest risk in the northern part of the study area, and far lower risk in the southern part. These findings suggest that risk mapping, and hence spatial targeting of hookworm mono-infections, is indeed useful at the spatial scale of the current investigation.

Apart from the fact that the outcome variables are different between hookworm mono-infections and single infections, the disparity between the maps could partly be explained by elevation being considerably more influential on a hookworm mono-infection $(\mathrm{RRR}=2.08)$ as suggested by the multinomial model rather than a hookworm single infection (odds ratio $(\mathrm{OR})=1.66$ ) estimated by a logistic regression model. Elevation may influence the occurrence of hookworm infections as follows. The northern, mountainous part of the study area experiences a higher average precipitation, which may positively influence the development of hookworm larvae in the soil. Combined with the presence of tropical forests, which might protect the contaminated soils from being washed out by heavy rains, these environmental features might create more suitable conditions for the development of infective hookworm larvae. With regard to $S$. mansoni, the mono-infection and the single infection risk maps were quite similar, with the exception that the highest mono-infection prevalence was found in the central-western part, whereas the highest single infections risk was found in the southwestern part.

It has been estimated that delivering a package of four drugs, i.e. albendazole (or mebendazole), praziquantel, ivermectin (or diethylcarbamazine), and azithromycine in areas where soil-transmitted helminthiasis, schistosomiasis, lymphatic filariasis, ochocerciasis and trachoma prevail, could save up to $47 \%$ of costs compared to non-integrative control programmes (Brady et al., 2006). Although targeting of multiple, neglected tropical diseases simultaneously remains a formidable challenge (Richards et al., 2006; Hotez et al., 2007; Kolaczinski et al., 2007), the presented mono-infection prediction, together with the prediction of single and co-infection prevalences, provides a useful tool for integrated and rapid risk mapping of the neglected tropical diseases. Using a combination of such models can in turn further and substantially enhance cost-effectiveness, and hence the sustainability of current and future integrated control programmes that are aimed at mass drug administration to reduce morbidity. Once the goal of morbidity control has been achieved, more sustainable control measures must be envisaged and implemented with some rigor, so that endless cycles of de-worming and re-worming can be avoided (Utzinger et al., 2003; Singer and Castro, 2007). Intervention packages consisting of sound information, education and communication, water supply and sanitation for preventing helminth infections, coupled with targeted chemotherapy and vaccines when they become available, that are readily adapted to the local eco-epidemiological and socio-cultural settings, are then needed and mapping can help to tailor such needs. 


\section{Acknowledgements}

This investigation received financial support from the Novartis Foundation, the Roche Research Foundation and the University of Queensland through fellowships to G. Raso, and the Swiss National Science Foundation supporting G. Raso (project no. PBBSB-109011), P. Vounatsou (325200-118379) and J. Utzinger (PP00B-102883).

\section{References}

Allen AVH, Ridley DS, 1970. Further observations on the formol-ether concentration technique for faecal parasites. J Clin Pathol 23, 545-546.

Basáñez MG, Marshall C, Carabin H, Gyorkos T, Joseph L, 2004. Bayesian statistics for parasitologists. Trends Parasitol 20, 85-91.

Beck-Wörner C, Raso G, Vounatsou P, N'Goran EK, Rigo G, Parlow E, Utzinger J, 2007. Bayesian spatial risk prediction of Schistosoma mansoni infection in western Côte d'Ivoire using a remotely-sensed digital elevation model. Am J Trop Med Hyg 76, 956-963.

Booth M, Vounatsou P, N'Goran EK, Tanner M, Utzinger J, 2003. The influence of sampling effort and the performance of the Kato-Katz technique in diagnosing Schistosoma mansoni and hookworm co-infections in rural Côte d'Ivoire. Parasitology 127, 525-531.

Brady MA, Hooper PJ, Ottesen EA, 2006. Projected benefits from integrating NTD programs in sub-Saharan Africa. Trends Parasitol 22, 285-291.

Brooker S, Clements ACA, Hotez PJ, Hay SI, Tatem AJ, Bundy DAP, Snow RW, 2006. The co-distribution of Plasmodium falciparum and hookworm among African schoolchildren. Malar J 5, 99.

Brooker S, Utzinger J, 2007. Integrated disease mapping in a polyparasitic world. Geospatial Health 1, 141-146.

Clements ACA, Lwambo NJS, Blair L, Nyandindi U, Kaatano G, Kinung'hi S, Webster JP, Fenwick A, Brooker S, 2006. Bayesian spatial analysis and disease mapping: tools to enhance planning and implementation of a schistosomiasis control programme in Tanzania. Trop Med Int Health 11, 490-503.

Cressie N, 1993. Regional mapping of incidence rates using spatial Bayesian models. Med Care 31, 60-65.
Diggle PJ, Tawn JA, Moyeed RA, 1998. Model-based geostatistics. J Roy Stat Soc C-App 47, 299-326.

Diggle PJ, Thomson MC, Christensen OF, Rowlingson B, Obsomer V, Gardon J, Wanji S, Takougang I, Enyong P, Kamgno J, Remme JH, Boussinesq M, Molyneux DH, 2007. Spatial modelling and the prediction of Loa loa risk: decision making under uncertainty. Ann Trop Med Parasitol 101, 499-509.

Engels D, Nahimana S, Gryseels B, 1996. Comparison of the direct faecal smear and two thick smear techniques for the diagnosis of intestinal parasitic infections. Trans $\mathrm{R}$ Soc Trop Med Hyg 90, 523-525.

Ezeamama AE, Friedman JF, Olveda RM, Acosta LP, Kurtis JD, Mor V, McGarvey ST, 2005. Functional significance of low-intensity polyparasite helminth infections in anemia. J Infect Dis 192, 2160-2170.

Fenwick A, 2006. New initiatives against Africa's worms. Trans R Soc Trop Med Hyg 100, 200-207.

Filmer D, Pritchett LH, 2001. Estimating wealth effects without expenditure data - or tears: an application to educational enrollments in states of India. Demography 38, 115-132.

Gelfand AE, Smith AFM, 1990. Sampling-based approaches to calculating marginal densities. J Am Stat Assoc 85, 398-410.

Gemperli A, Vounatsou P, Kleinschmidt I, Bagayoko M, Lengeler C, Smith T, 2004. Spatial patterns of infant mortality in Mali: the effect of malaria endemicity. Am J Epidemiol 159, 64-72.

Gosoniu L, Vounatsou P, Sogoba N, Smith T, 2006. Bayesian modelling of geostatistical malaria risk data. Geospatial Health 1, 127-139.

Hotez PJ, Molyneux DH, Fenwick A, Kumaresan J, Ehrlich Sachs S, Sachs JD, Savioli L, 2007. Control of neglected tropical diseases. N Engl J Med 357, 1018-1027.

Hotez PJ, Molyneux DH, Fenwick A, Ottesen E, Ehrlich Sachs S, Sachs JD, 2006. Incorporating a rapid-impact package for neglected tropical diseases with programs for HIV/AIDS, tuberculosis, and malaria. PLoS Med 3, e102.

Kabatereine NB, Tukahebwa E, Kazibwe F, Namwangye H, Zaramba S, Brooker S, Stothard JR, Kamenka C, Whawell S, Webster JP, Fenwick A, 2006. Progress towards countrywide control of schistosomiasis and soil-transmitted helminthiasis in Uganda. Trans R Soc Trop Med Hyg 100, 
208-215.

Katz N, Chaves A, Pellegrino J, 1972. A simple device for quantitative stool thick-smear technique in schistosomiasis mansoni. Rev Inst Med Trop Sao Paulo 14, 397-400.

Kazembe LN, Appleton CC, Kleinschmidt I, 2007. Spatial analysis of the relationship between early childhood mortality and malaria endemicity in Malawi. Geospatial Health 2, 41-50.

Keiser J, N'Goran EK, Traoré M, Lohourignon KL, Singer BH, Lengeler C, Tanner M, Utzinger J, 2002. Polyparasitism with Schistosoma mansoni, geohelminths, and intestinal protozoa in rural Côte d'Ivoire. J Parasitol $88,461-466$.

Kolaczinski JH, Kabatereine NB, Onapa AW, Ndyomugyenyi R, Kakembo AS, Brooker S, 2007. Neglected tropical diseases in Uganda: the prospect and challenge of integrated control. Trends Parasitol 23, 485-493.

Lammie PJ, Fenwick A, Utzinger J, 2006. A blueprint for success: integration of neglected tropical disease control programmes. Trends Parasitol 22, 313-321.

Marti HP, Escher E, 1990. SAF - eine alternative Fixierlösung für parasitologische Stuhluntersuchungen. Schweiz Med Wochenschr 120, 1473-1476.

Molyneux DH, Hotez PJ, Fenwick A, 2005. "Rapid-impact interventions": how a policy of integrated control for Africa's neglected tropical diseases could benefit the poor. PLoS Med 2, e336.

Raso G, Luginbühl A, Adjoua CA, Tian-Bi NT, Silue KD, Matthys B, Vounatsou P, Wang Y, Dumas ME, Holmes E, Singer BH, Tanner M, N'Goran EK, Utzinger J, 2004a. Multiple parasite infections and their relationship to selfreported morbidity indicators in a community of rural Côte d'Ivoire. Int J Epidemiol 33, 1092-1102.

Raso G, Matthys B, N'Goran EK, Tanner M, Vounatsou P, Utzinger J, 2005a. Spatial risk prediction and mapping of Schistosoma mansoni infections among schoolchildren living in western Côte d'Ivoire. Parasitology 131, 97-108.

Raso G, N'Goran EK, Toty A, Luginbühl A, Adjoua CA, Tian-Bi NT, Bogoch II, Vounatsou P, Tanner M, Utzinger J, 2004b. Efficacy and side effects of praziquantel against Schistosoma mansoni in a community of western Côte d'Ivoire. Trans R Soc Trop Med Hyg 98, 18-27.
Raso G, Utzinger J, Silué KD, Ouattara M, Yapi A, Toty A, Matthys B, Vounatsou P, Tanner M, N'Goran EK, 2005b. Disparities in parasitic infections, perceived ill health and access to health care among poorer and less poor schoolchildren of rural Côte d'Ivoire. Trop Med Int Health 10, 42-57.

Raso G, Vounatsou P, Gosoniu L, Tanner M, N'Goran EK, Utzinger J, 2006a. Risk factors and spatial patterns of hookworm infection among schoolchildren in a rural area of western Côte d'Ivoire. Int J Parasitol 36, 201-210.

Raso G, Vounatsou P, Singer BH, N'Goran EK, Tanner M, Utzinger J, 2006b. An integrated approach for risk profiling and spatial prediction of Schistosoma mansoni-hookworm coinfection. Proc Natl Acad Sci USA 103, 6934-6939.

Richards FOJ, Eigege A, Miri ES, Jinadu MY, Hopkins DR, 2006. Integration of mass drug administration programmes in Nigeria: the challenge of schistosomiasis. Bull World Health Organ 84, 673-676.

Singer BH, Castro MC, 2007. Bridges to sustainable tropical health. Proc Natl Acad Sci USA 104, 16038-16043.

Utzinger J, Bergquist R, Xiao SH, Singer BH, Tanner M, 2003. Sustainable schistosomiasis control - the way forward. Lancet 362, 1932-1934.

Utzinger J, Booth M, N'Goran EK, Müller I, Tanner M, Lengeler C, 2001. Relative contribution of day-to-day and intra-specimen variation in faecal egg counts of Schistosoma mansoni before and after treatment with praziquantel. Parasitology 122, 537-544.

Utzinger J, de Savigny D, 2006. Control of neglected tropical diseases: integrated chemotherapy and beyond. PLoS Med 3, e112.

Utzinger J, N'Goran EK, Marti HP, Tanner M, Lengeler C, 1999. Intestinal amoebiasis, giardiasis and geohelminthiases: their association with other intestinal parasites and reported intestinal symptoms. Trans R Soc Trop Med Hyg 93, 137-141.

WHO, 2002. Prevention and control of schistosomiasis and soil-transmitted helminthiasis: report of a WHO expert committee. WHO Tech Rep Ser No 912.

WHO, 2006. Preventive chemotherapy in human helminthiasis: coordinated use of anthelminthic drugs in control interventions: a manual for health professionals and programme managers. 